\title{
Dietary phylloquinone intake in young British Caucasian women: influences on indices of bone health
}

\author{
L. A. Davey ${ }^{1}$, S. A. Sheffield ${ }^{1}$, A. D. Woolf ${ }^{2}$ and S. A. Lanham-New ${ }^{1}$ \\ ${ }^{1}$ Nutritional Sciences Division, Faculty of Health and Medical Sciences, University of Surrey, Guildford GU2 7HX, UK \\ and ${ }^{2}$ Department of Medicine, University of Plymouth, Plymouth, UK
}

Attainment of maximum peak bone mass (PBM) is important for osteoporosis prevention. Vitamin $\mathrm{K}$ is a key nutrient for bone health. Vitamin $\mathrm{K}$ is a cofactor in the carboxylation of glutamate (Glu) residues to $\gamma$-carboxyglutamyl or 'Gla' residues in specific proteins (vitamin K-dependent proteins) $^{(1)}$. Observational studies have demonstrated an association between vitamin $\mathrm{K}$ intake and bone health although few have focused on young women ${ }^{(2)}$. Vitamin $\mathrm{K}$ is found in two main forms: phylloquinone (formerly vitamin $\mathrm{K}_{1}$; found in green leafy vegetables and plant oils); menaquinones (formerly vitamin $\mathrm{K}_{2}$; synthesised by intestinal bacteria and found in fermented foods). There is no reference nutrient intake for vitamin $\mathrm{K}$ intake but recommendations are based on safe levels for blood coagulation and are set at $1 \mu \mathrm{g} / \mathrm{kg}$ body weight per $\mathrm{d}^{(3)}$.

The aims of the present investigation were threefold: (1) to compare phylloquinone intakes with recommended levels; (2) to assess the daily variation in phylloquinone intakes; (3) to explore associations between phylloquinone intakes and indices of bone health in the study population.

A total of 275 British Caucasian women aged 20-30 years were recruited from Cornwall and Surrey. Dual X-ray absorptiometry (DXA) was used to assess bone mineral density (BMD) at the lumbar spine (LS), femoral neck and whole body (WB; Surrey only). Other measurements included a blood and urine sample collection, anthropometry, general lifestyle characteristics and a $7 \mathrm{~d}$ food diary. Dietary analysis was completed on 147 of the records using Microdiet Version 2 (Downlee Systems Ltd, Chapel-en-le-Frith, Derbyshire, UK).

\begin{tabular}{|c|c|c|c|c|c|c|}
\hline & \multicolumn{6}{|c|}{ Phylloquinone intake $(\mu \mathrm{g})$} \\
\hline & Overall & Day 1 & Day 2 & Day 3 & Day 4 & Day 5 \\
\hline $\bar{n}$ & 147 & 79 & 79 & 79 & 79 & 79 \\
\hline Mean & 30.0 & 20.9 & 33.7 & 31.9 & 20.0 & 29.5 \\
\hline $\mathrm{SD}$ & 35.1 & 33.0 & 71.6 & 60.1 & 27.5 & 70.4 \\
\hline Minimum & 0.82 & 0 & 0 & 0 & 0 & 0 \\
\hline Maximum & 255 & 182 & 406 & 316 & 136 & 556 \\
\hline
\end{tabular}

The mean weight of the participants was $66 \mathrm{~kg}$, therefore the mean intake of phylloquinone should be approximately $66 \mu \mathrm{g} / \mathrm{d}$. The Table shows that the mean intake of phylloquinone was well below recommended levels, with $91 \%$ of the participants not reaching the recommended safe intake level. There was also variation in daily intakes of phylloquinone. The data demonstrated weak associations between phylloquinone intake and indices of bone health. Trends were established between mean phylloquinone intake and BMD at the LS $(P=0.092)$ and the WB $(P=0.081)$. The highest BMD measurements were seen in the highest tertiles and quartiles of phylloquinone intake. A trend towards a difference in the mean intake was found when comparing LS BMD in the different tertiles and quartiles. When comparing phylloquinone intake in those participants with normal BMD to those with a low BMD a significantly lower intake of phylloquinone was seen in those participants with a low WB BMD $(P=0.016)$, as well as a trend towards lower phylloquinone intakes in those with a low BMD at the femoral neck $(P=0.092)$. A large percentage of young British women may have inadequate phylloquinone intakes that do not reach the recommended safe level needed for blood coagulation. Further work is required to establish the relationship between vitamin $\mathrm{K}$ and peak bone mass attainment in the young adults.

The Young British Women's Study was funded by the National Osteoporosis Study.

1. Szulc P, Chapuy M-C, Meunier P et al. (1993) J Clin Invest 91, 1769-1774.

2. Booth SL (2003) Am J Clin Nutr 77, 512-516.

3. Department of Health (1991) Dietary Reference Values for Food, Energy and Nutrition for the United Kingdom. London: H. M. Stationery Office. 